\title{
GRAIDs: a framework for closing the gap in the availability of health promotion programs and interventions for people with disabilities
}

\author{
James H Rimmer ${ }^{1 *}$, Kerri A Vanderbom', Linda G Bandini ${ }^{2,3}$, Charles E Drum ${ }^{4}$, Karen Luken ${ }^{5}$,
}

Yolanda Suarez-Balcazar ${ }^{6}$ and lan D Graham ${ }^{7}$

\begin{abstract}
Background: Evidence-based health promotion programs developed and tested in the general population typically exclude people with disabilities. To address this gap, a set of methods and criteria were created to adapt evidence-based health promotion programs for people with disabilities. In this first study, we describe a framework for adapting evidence-based obesity prevention strategies for people with disabilities. We illustrate how the framework has been used to adapt the U.S. Centers for Disease Control and Prevention's (CDC) obesity prevention strategies for individuals with physical and developmental disabilities.
\end{abstract}

Methods: The development of inclusion guidelines, recommendations and adaptations for obesity prevention (referred to as GRAIDs - Guidelines, Recommendations, Adaptations Including Disability) consists of five components: (i) a scoping review of the published and grey literature; (ii) an expert workgroup composed of nationally recognized leaders in disability and health promotion who review, discuss and modify the scoping review materials and develop the content into draft GRAIDs; (iii) focus groups with individuals with disabilities and their family members (conducted separately) who provide input on the potential applicability of the proposed GRAIDs in real world settings; (iv) a national consensus meeting with 21 expert panel members who review and vote on a final set of GRAIDs; and (v) an independent peer review of GRAIDs by national leaders from key disability organizations and professional groups through an online web portal.

Results: This is an ongoing project, and to date, the process has been used to develop 11 GRAIDs to coincide with 11 of the 24 CDC obesity prevention strategies.

Conclusion: A set of methods and criteria have been developed to allow researchers, practitioners and government agencies to promote inclusive health promotion guidelines, strategies and practices for people with disabilities. Evidence-based programs developed for people without disabilities can now be adapted for people with disabilities using the GRAIDs framework.

Keywords: Disability, Health promotion, Guideline adaptation, Obesity

\section{Background}

People with disabilities face challenges in acquiring health behaviors critical to the optimization of their own selfmanaged health and wellness. Children with disabilities are much less likely to participate in school-based or community-based health promotion activities with their

\footnotetext{
* Correspondence: jrimmer@uab.edu

${ }^{1}$ University of Alabama at Birmingham/Lakeshore Foundation Research

Collaborative, 4000 Ridgeway Dr., Birmingham, AL 35209, USA

Full list of author information is available at the end of the article
}

peers, and are far more likely to be sedentary and have a poorer diet quality [1-4]. Adults and youth with disabilities have higher rates of secondary health conditions including pain, fatigue, depression, and obesity [5-8], and report lower rates of participation in health promoting activities such as physical activity $[9,10]$. Despite physical activity and good nutrition being the cornerstones of evidencebased health promotion interventions for reducing the risk of comorbidities (e.g., diabetes, heart disease, stroke) [11], many people with disabilities or caregivers who have 
a child with a disability experience substantial difficulty accessing these programs $[12,13]$. Inaccessible facilities, lack of transportation to and from indoor and outdoor recreation venues, absence of knowledgeable staff who understand how to include people with disabilities in their programs, and a general perception/attitude among providers that people with disabilities need 'specialized' vs. integrated services, feed into a culture of isolation and separation [14-16] and exposes this population to disproportionately higher rates of health disparities [17].

Not surprisingly, the wealth of evidence-based health promotion literature in policy, environmental, programmatic and infrastructure changes has been generated by researchers who unintentionally or unknowingly excluded people with disabilities [18]. The vast majority of health promotion research targets populations who do not have a disability, and researchers often, if not always, use a 'preexisting condition' or disability as one of its exclusion criteria, thus limiting the generalizability of these findings for people with disabilities [19]. While specialized health promotion programs for certain disability groups can be quite valuable in the short-term, they are often difficult to sustain after the project ends and the resources are no longer available (e.g., staff, time, transportation, expertise, space).

The adoption of certain evidence-based research findings often takes years, if not decades, to reach its endpoint in clinical or community practice [20]. The Institute of Medicine [21], the Canadian Partnership Against Cancer (CAN-IMPLEMENT) [22,23], and the Guidelines International Network $[24,25]$ have suggested that unnecessary and costly duplication of programs or services could be minimized or avoided if evidence-based guidelines were adapted rather than developed de novo. An alternative to the development of health promotion interventions designed for select groups of people with or without disabilities is to formalize a process for adapting guidelines established from the best available evidence. The Guidelines International Network defines adaptation as 'the systematic approach to the endorsement and/or modification of a guideline(s) produced in one cultural and organizational setting for application in a different context' [24]. Adaptation can render a program more responsive to a particular target group and increase the program's sensitivity and fit for a new population that was not part of the original research [26]. It also offers greater transferability to real world settings (i.e., knowledge translation) because the adaptations can be selected by local service providers based on the needs of the end user(s) and the local context [23].

Adapting evidenced-based strategies/programs established on people without disabilities for people with disabilities holds strong potential for accelerating use of existing and new evidence-based findings in this underrepresented population. This paper describes the development of a process that includes a set of methods and criteria for adapting evidence-based health promotion guidelines for people with disabilities. For the purpose of this paper, we illustrate the approach using a set of evidence-based obesity prevention strategies developed by the U. S. Centers for Disease Control and Prevention (CDC) [27] that have been adapted for youth and young adults with physical and developmental disabilities.

\section{Methods}

\section{Procedures}

The comprehensive set of methods and criteria used in this study was modeled after the Guidelines International Network ADAPTE framework [24]. This framework was chosen because it formalizes a set of methods for adapting evidence-based guidelines developed in one context for use in a different context without diminishing the quality of the original guideline(s). The end product was a set of GRAIDs, which stands for Guidelines, Recommendations, and Adaptations Including Disability.

\section{Guideline adaptation conceptual framework}

Producing high quality inclusive guidelines, recommendations and adaptations to effect change in public health practice requires strong procedural fidelity. As such, the conceptual framework shown in Figure 1 was developed to formalize a process that would endure the scrutiny of researchers, practitioners, and federal and public health officials. The framework was established with an internationally recognized scholar in guideline adaptation (I. Graham). A series of in-person and online meetings were arranged to complete and finalize the set of decision algorithms that were subsequently used to develop the GRAIDs.

\section{Phase I. Set-up \\ Phase I, Step 1. Identification of evidence-based obesity prevention strategies}

The first step in the health promotion guideline/strategy adaptation process was to identify existing guidelines/ strategies that were appropriate to adapt for the target population. For our example, the CDC's 24 evidence-based obesity prevention strategies from the published report, 'Recommended Community Strategies and Measurements to Prevent Obesity in the United States, Implementation and Measurement Guide' [27] were selected. The strategies were based on extensive systematic reviews of the best available evidence and are currently recommended by the U.S. Department of Health and Human Services (HHS) and CDC as the major evidence-based strategies for reducing obesity in the U.S. population (children, adolescents and adults). The 24 evidence-based strategies, shown in Additional file 1: Table S1, are being used across the U.S. to support health promotion/disease prevention programs targeting policy, systems and environmental changes at the 


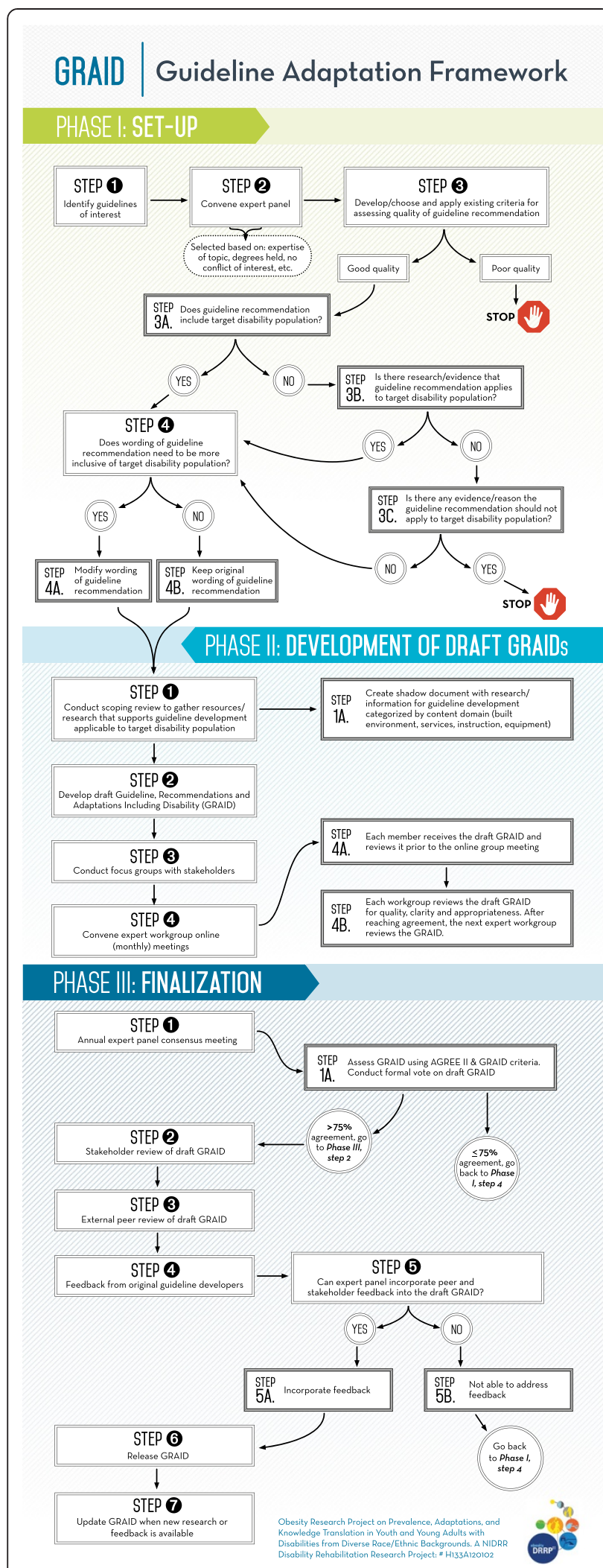

Figure 1 GRAID guideline adaptation framework. community level [28]. Because of the rigor of development of the CDC evidence-based strategies, we did not feel the need to formally assess their quality before proceeding with their adaptation. When the quality of source guidelines is not known or suspect, they should be quality approved using a validated guideline appraisal instrument such as the AGREE II instrument (see below) to ensure that they are of sufficient quality to justify adapting them.

\section{Phase I, Step 2. Formation of expert panel/workgroup}

The next step in the guideline/strategy adaptation process was the formation of an expert panel composed of highly qualified professionals and researchers who were appropriate content experts in obesity prevention/management and disability. They were recruited using the following criteria: (i) doctoral-level degree and/or strong clinical/public health background associated with disability and health; (ii) previous experience in health promotion/weight management interventions and programs for people with disabilities; (iii) involved in a clinical setting and/or conducting research across interventions related to obesity in people with disabilities; (iv) expertise in at least one of the four content areas (nutrition, physical activity, cultural competency, and policy); and (v) no conflict of interest (COI) related to the development of adapted guidelines by having a competing project or grant.

The process for identifying and selecting expert panel members was completed after an extensive review of the literature to identify content experts who had published in the field, in addition to contacting hospitals, universities and rehabilitation centers where work was being completed by recognized experts in health, nutrition and disability. A total of 21 experts were selected from across the U.S. representing all geographic regions. The expert panel was subdivided into four workgroups by content area specialty, and each workgroup was composed of one chair and four to five members (described in Phase II, Step 4).

\section{Phase I, Step 3. Criteria for creating quality GRAIDs}

Developing a quality set of adapted guidelines required a formal process for capturing key elements in its design and implementation. Several researchers have noted that there are numerous factors that could influence the development of a quality guideline, including the criteria used to establish the guideline $[23,29,30]$. At the initiation of the project, the expert panel met to develop the criteria that would be used for guideline development and finalization of the GRAIDS. The expert panel reviewed the 23 AGREE II criteria [31] (Additional file 2: Table S2) and came up with 15 additional criteria (Additional file 3: Table S3) that were associated with disability-specific relevance to adapted guideline development methodology. The six AGREE II constructs are: Scope and Purpose (three items), Stakeholder Involvement (three items), 
Rigor of Development (eight items), Clarity and Presentation (three items), Applicability (four items), and Editorial Independence (two items).

The combined set of criteria considers the quality of a guideline based on the level of confidence that can be placed in the process, including the minimization of biases linked to the rigor of development, presentation and applicability of a clinical practice guideline, clear reporting of each step in the development process, and the guideline's applicability to people with disabilities. The additional set of 15 criteria relate to the appropriateness (e.g., age, gender, culture), usability (e.g., product, service or program), and acceptability (e.g., can be implemented without drawing negative attention to the end user) of each GRAID with respect to people with disabilities. The GRAID criteria were voted on and approved by the 21-member panel, providing a level of expert validity. The expert panel used these 38 criteria when they voted on final acceptance of each GRAID.

\section{Phase II. Development of draft GRAIDs \\ Phase II, Step 1. Structural elements of content development}

Each GRAID consists of three structural elements: (i) an overarching inclusion guideline that matches the CDC strategy; (ii) a set of inclusion recommendations and adaptations separated into one of four content domains (Built Environment, Services, Instruction, and Equipment); and (iii) a set of policy recommendations. 'Adaptations' provided the finest level of detail for supporting the inclusion of an individual into an existing health promotion program and contained a variety of options so that implementation strategies could be customized for the end user at the local level. This information was categorized under an overarching 'Inclusion Recommendation' for each content domain. The four sets of 'Inclusion Recommendations' provided guidance at the administrative/programmatic level on how a specific set of adaptations could be incorporated into an existing health promotion program or service. A separate set of 'Policy Recommendations' were also included with each GRAID that referenced any existing federal law requiring access to certain services or programs, or any recommendations that had the potential to change practice or modify existing policy at the local or state level. After the adaptations and inclusion and policy recommendations were drafted, the 'inclusion guideline' was written and linked to the specific CDC strategy. The GRAIDs were then reviewed for cultural competency and modifications made where necessary. A sample GRAID is shown in Additional file 4: Table S4.

\section{Phase II, Step 2. Data sources for creating the GRAID}

Several different data sources were used to create each GRAID. Since there were few empirical studies that addressed adaptations associated with physical activity and nutrition for individuals with disabilities, we relied not only on scholarly articles but on textbooks, dissertations, theses, reports, online media, and the expertise of staff to review the literature related to obesity prevention. Three trained reviewers searched for information using keywords related to each of the CDC obesity strategies. Key words varied depending on the focus of the strategy. For example, in GRAID 8, Increasing Access to Outdoor Recreation, keywords included 'communities AND recreation AND disabilities,' 'communities AND outdoor OR recreation AND disabilities,' 'obesity AND parks,' 'outdoor recreation AND accessibility.' Various databases were also searched (e.g., PubMed, SportDiscus, CINAHL, Scopus, dissertation abstracts). Reviewer materials were regularly assessed by the coordinator of the project for quality, content, currency and appropriateness.

Prior to conducting the first review, the reviewers were required to practice the process and reach at least $80 \%$ inter-rater reliability choosing information from the article and categorizing it into one of the four content domains: Built Environment, Services, Instruction and Equipment. Legislative materials relevant to the draft GRAID were placed in a separate file and labeled, Policy. A total of $20 \%$ of articles in a domain were randomly chosen. Each reviewer had to read through each article and identify the relevant information that could be used as an adaptation or recommendation and categorize it into the correct domain. Once $80 \%$ inter-rater reliability was reached, the three reviewers were able to complete their review of the literature. Content was placed in an Excel file referred to as the GRAID 'Shadow Document' that included the name of the author(s), source of materials (e.g., peerreviewed article, book, website), and date of publication. The material was then organized by content domain and used by the expert workgroup to create the draft GRAIDs.

\section{Phase II, Step 3. Focus groups with stakeholders}

A major emphasis in the disability community is providing opportunities for people with disabilities and family members to have a voice in the development of programs and materials related to their health and wellbeing [32,33]. It is important to have a clear understanding of the problem from the perspective of the target population. One method for engaging end users in the GRAID development process was through a series of focus groups conducted on a specific topic area at different stages of the research process. For example, five focus groups were conducted with youth/adults with disabilities and their family members in three different U.S. states. Institutional review board approval and consent/assent by each participant were obtained prior to conducting the focus groups. The focus groups for youth/adults with disabilities were held separately from the focus groups 
for family members/caregivers. Focus group participants had to have the ability to respond to a series of questions and were paid a nominal fee for their time (\$25). Each focus group had between 4 and 10 participants and was conducted in either English or Spanish to ensure cultural representation. The focus groups lasted approximately one hour and involved the researcher asking a series of semi-structured, open-ended questions about behaviors, experiences, barriers and facilitators related to physical activity and nutrition. The focus groups were audio recorded, transcribed and analyzed by a team of researchers and stakeholders. Findings from the focus groups resulted in the development of several new adaptations, but also validated that the adaptations identified and developed from the review of literature and expert workgroup members were consistent with their comments.

\section{Phase II, Step 4. Expert workgroup online meetings}

As described in the CAN-IMPLEMENT guide and Knowledge-To-Action framework, forming working panels is necessary to help oversee the adaptation process [22]. In the current study, the expert panel was subdivided into four expert workgroups to oversee the guideline adaptation process. The four expert workgroups (Physical Activity, Nutrition, Cultural Competency, Policy) were convened through an internet-based online meeting every month for two hours to review the content that the scoping reviewers identified as applicable to one of the CDC evidence-based strategies (one strategy is reviewed per online meeting). The workgroup assignments were staggered to allow the primary content experts in Physical Activity and Nutrition to conduct their review first, followed by the Cultural Competency workgroup. If the CDC strategy related to physical activity, the Physical Activity Expert Workgroup met first followed by the Nutrition Expert Workgroup. If the strategy related to nutrition, then the expert workgroups met in reverse order. The second workgroup addressed any gaps in the draft GRAID developed by the primary workgroup.

After the Physical Activity and Nutrition Workgroups completed their review, the Cultural Competency Workgroup met online to review the draft GRAID for cultural relevance. Cultural competency in this study referred to the applicability of the strategy to the norms, values and beliefs of ethnically and racially diverse (e.g., African American and Latino) youth and adults with disabilities [34]. A culturally competent intervention met the following criteria: the intervention was based on the cultural values of the target group; the strategies that made up the intervention reflected the subjective cultural characteristics, including interests, expectations, and norms of members of diverse race and ethnic groups; and the components that made up the strategies reflected the behavioral preferences and expectations of the members of the target group
[34,35]. The five Cultural Competency workgroup members had backgrounds in race/ethnic diversity and were selected because of their combined expertise in cultural competency, and disability and health promotion.

Finally, after the three workgroups completed their work on the draft GRAID, the Policy Workgroup convened, reviewed the draft GRAID, and formulated a set of policy recommendations. Whenever possible, a policy recommendation used federal or state law to support the recommendation.

\section{Preparation for online workgroup meetings}

Prior to the online meeting, the coordinator reviewed the materials identified by the scoping review team to ensure that they were appropriate for guideline adaptation. The Expert Workgroup received the materials several days in advance of the meeting, and all members were expected to review them prior to convening. The expert workgroup also received a glossary of terms (Additional file 5: Table S5) so that each member stated a new guideline, recommendation or adaptation with consistent terminology. The group discussion involved a review of the materials in a structured, orderly process by content domain (built environment, services, instruction, equipment). The material was posted on an online meeting platform so that each member was able to view the same content. The coordinator moderated the meeting and responded to questions from workgroup members. One scoping reviewer was always present in case there were questions or if clarification was needed related to the primary source of the content. During the discussion, each workgroup member could agree or disagree about the quality, clarity or appropriateness of any of the content. If the workgroup agreed that the statement was appropriate, it was converted into a draft adaptation under the appropriate content domain. The GRAID was then reviewed by the next Expert Workgroup. The process for the online workgroup meetings became much more efficient after the first few draft GRAIDs were developed.

\section{Phase III. Finalization}

\section{Phase III, Step 1. Annual expert panel consensus meeting}

The four expert workgroups were convened into a 21member expert panel that met at a remote location for 2 days to review all of the GRAIDs that were developed in the previous 12 months. Expert panel members were divided into two groups. Each group consisted of 10 to 11 members and was assigned two domains (e.g., built environment and equipment). An effective and neutral facilitator explained the procedures and rules and then oversaw the process to ensure that there was a balanced contribution from all members. As recommended by G-I-N [36], the facilitator was not a content expert and remained objective and unbiased in the finalization of 
the draft GRAIDs. This was done to reduce the potential introduction of personal bias that might arise with a facilitator who has expertise in the content area being discussed.

Panel members provided feedback on each GRAID (e.g., content, wording, structure). If at least one member disagreed with the wording or quality of any element of the GRAID, the group discussed the item until it was resolved. If the issue could not be resolved by the expert panel, the facilitator entered the discussion to help clarify the issue and settle any disagreement.

After the consensus meeting, all recommended changes were added to each GRAID and then voted on anonymously by the entire expert panel using a web-based application. The expert panel used the AGREE II and GRAID criteria for evaluating each GRAID prior to voting. There had to be $75 \%$ agreement among the expert panel members for a GRAID to be finalized during this phase.

\section{Phase III, Step 2. Stakeholder review of draft GRAID}

Stakeholders also reviewed each GRAID prior to finalization. A web application was created for stakeholder comment on the draft GRAID. This provided an opportunity for the greatest number of invited stakeholders to contribute comments about the posted GRAID. The web application captured feedback and commentary regarding each GRAID including:

1. Applicability - across target population or limitations by age, gender, and/or cultural groups;

2. Content Analysis - Physical Activity (adaptations categorized under the Built Environment, Services, Instruction, and Equipment); Nutrition (same as Physical Activity); Cultural (cultural adaptations to physical activity and nutrition appropriate for minority groups); and Policy (policy-related recommendations using federal, state or city ordinances to support, whenever possible, an adaptation/recommendation);

3. Potential Barriers and Facilitators to implementation;

4. Reference information for adding or modifying content. If no reference was available, the item had to provide basic information on name, location and contact for the program in which it is or was used;

5. Contact information including at least a valid email address so project staff can follow up if there are additional questions.

\section{Phase III, Step 3. External peer review of draft GRAID}

The draft GRAIDs were posted to a secure website and an independent external peer review group that had no involvement in developing the GRAID was solicited to review each one. The external peer review group was composed of four to six professionals with expertise in disability and health. Each member reviewed the GRAID for content, clarity and consistency using the AGREE II [31] and GRAID criteria. The peer reviewers also had the opportunity to provide written feedback.

All comments from the stakeholder and peer review were disseminated to the expert panel. Major issues or concerns were discussed through an online meeting forum, and any recommended changes to a GRAID required a $2 / 3$ majority vote by the expert panel.

\section{Phase III, Step 4. Feedback from original guideline developers}

The final set of GRAIDs was sent back to the original guideline development group, which in the current project was the Division of Nutrition, Physical Activity and Obesity at the CDC. To facilitate this process, we included a CDC program officer as a consultant on the project who attended the annual consensus meeting to gain a better understanding of the process and to offer guidance, when necessary, on the appropriate terminology to use when discussing each GRAID and to ensure that the adaptations were consistent with the original CDC strategy.

\section{Phase III, Step 5. Incorporate peer and stakeholder feedback} Prior to releasing the GRAID, one final step involves the review of external stakeholder feedback. Any critical information that may have been missed by the expert panel is incorporated into the final GRAID prior to release.

\section{Phase III, Step 6. Release of the guideline}

The plan is to release the GRAIDs through an extensive dissemination network that includes several organizational partners associated with disability, health, and the health promotion area (i.e., obesity). Many of these organizations wrote a letter of support when the project was funded indicating their commitment to disseminating the GRAIDs through their membership and affiliates.

\section{Discussion}

The major aim of this project was to develop a set of methods and criteria that would facilitate the inclusion of people with disabilities in mainstreamed health promotion research and programs. The GRAID framework provides researchers, practitioners, and funding agencies with a structured, systematic process for adapting evidencedbased health promotion guidelines developed in the general (non-disabled) population for people with disabilities. Adapting existing evidence-based guidelines/ programs vs. de novo guideline/program development provides a unique opportunity for researchers and programmers in health promotion and disability to work 
closely with health promotion researchers and programmers who have no expertise in disability to establish a unifying inclusion science framework that represents the needs of all research participants/community members.

The uniqueness of the GRAID framework is that it incorporates both traditional (i.e., scoping reviews, consensus panel) and non-traditional (i.e., focus groups with individuals with disabilities and/or family members, stakeholder feedback through an open source web-based portal) data in its development. Each GRAID consists of multiple adaptations to allow implementers or researchers to select the appropriate set of inclusion elements that coincide with their organizational, structural, economic and cultural context.

Feedback from the expert panel about the GRAID development process has been favorable. Many panel members expressed enthusiasm about using the GRAIDs in their own research and/or practice. The regular participation in the monthly phone calls and high attendance rates at the annual consensus meeting were reflective of their support for the process. Several panel members commented that while the GRAID framework required substantive support and facilitation to reach finalization, it was an extremely worthwhile process and holds promise for promoting integration of people with disabilities in existing and future health promotion research and programs.

The current state-of-the-science in health promotion research generally runs on two parallel tracks: one designed for the general population where most of the resources are directed, and the other track for disabilityspecific research with significantly less resources. The failure of these two systems to capture the best elements from both sets of prevention programs and infuse them into existing networks, systems and environments to effectively promote the health of all community members results in gaps and disparities in health promotion access, inefficient use of time and money, and health inequities (a lower impact on health outcomes for people with disabilities).

The complexity of changing the behaviors of organizations and service providers warrants a paradigm shift away from isolated interventions with specific disability groups toward the inclusion of individuals with disabilities in existing evidence-based interventions and programs available today and in the future. Given limitations in government resources and the difficulty in establishing randomized controlled trials with small subsets of the population (e.g., a certain type of disability) [37], funding agencies should collaborate on developing an implementation science framework that encourages researchers to examine the usefulness/value of the GRAIDs, how they are implemented in the community and their potential impact on health outcomes in the appropriate target group. The GRAID methodology attempts to systematically bridge science and practice and presents a framework for promoting the inclusion of people with disabilities in existing and future health promotion research and programs. It is our hope that this new framework will lay the foundation for an effective and sustainable approach to guideline adaptation of existing evidence-based health promotion programs and future research directives developed for the general population for people with disabilities.

\section{Limitations}

Given the limited research and information related to adapting evidence-based health promotion strategies for people with disabilities, much of the content used to formulate the GRAIDs was derived from the non-peer reviewed literature in the form of books, articles, papers, reports and websites. Only English literature was reviewed. It is plausible that we may have missed a certain adaptation for a specific disability group. Thus far, the GRAIDs have not been empirically tested. Future research is needed to test the effectiveness of the GRAIDs in real world, inclusive health promotion settings.

\section{Plans for GRAID Validation}

The GRAIDs developed in this study provide an opportunity for researchers and practitioners to test their validity with certain target groups of people with disabilities who were not part of the original evidence-based research. Using integrated knowledge translation, the GRAIDs can be tested at many different levels including national, state or community initiatives, existing or new communitybased 'best practice' or evidence-based programs, or at the policy level to support inclusive health promotion practices and services for people with disabilities. Scale up evaluation of GRAIDs in existing and future evidencebased health promotion programs will help clarify their potential as a means for promoting 'real world' inclusive health promotion practices. Funding agencies may consider using the GRAID methodology and/or currently existing GRAIDs to support research and evaluation efforts that examine their utility for children, adults and seniors with disabilities at the local, state or national level.

\section{Conclusion}

The GRAID framework promotes the inclusion of people with disabilities in current and future health promotion research and programs and is an important first step in establishing an inclusion science that recognizes the needs of people with disabilities within the same context as the rest of the community [38]. The high rates of health disparities reported among children [5,39] and adults with disabilities $[17,40]$ warrants this new approach for promoting uptake of successful evidence-based practices developed on non-disabled populations for people with disabilities. For long-term sustainable health improvements to occur, 
community planners, service providers, and health professionals need a common set of GRAIDs that facilitate the inclusion of people with disabilities in health promotion initiatives in schools, healthcare facilities, employment settings, and the community-at-large [32,41].

\section{Additional files}

Additional file 1: Table S1. Centers for Disease Control and Prevention 24 Evidence-Based Obesity Prevention Strategies.

Additional file 2: Table S2. AGREE II Criteria.

Additional file 3: Table S3. GRAID Criteria for Assessment of

Disability-Specific Methodological Quality.

Additional file 4: Table S4. Sample GRAID.

Additional file 5: Table S5. GRAID Glossary.

\section{Competing interests}

The authors declare that they have no competing interests.

\section{Authors' contributions}

JHR conceptualized the methodology and was the primary contributor to this manuscript. KAV assisted with the writing and coordinated the completion of the methodology. IDG was the primary consultant, advising on the steps and modification of the methodology to ensure fidelity and rigor of the evidence-base being adapted. IDG was also a key contributor to the content and editing of the manuscript. LGB, CED, KL, YSB also contributed to the editing of the manuscript. All authors read and approved the final manuscript.

\section{Acknowledgements}

A special thanks to our expert panel members who worked diligently on the development of the GRAIDs: Yolanda Suarez-Balcazar, University of Illinois at Chicago; Linda Bandini, Eunice Kennedy Shriver Center/UMass Medical School \& Boston University; Lynne Cory, Clemson University; Charles Drum, University of New Hampshire; Georgia Frey, Indiana University; Brigida Hernandez, YAI Network; Kristie Hubbard, Tufts University; Kathleen Humphries, Oregon Health and Science University; Willi Horner-Johnson, Oregon Health and Science University; June Isaacson Kailes, Harris Family Center for Disability \& Health Policy; Joanne Kouba, Loyola University Chicago; Karen Luken, Disability and Health consultant; Sandra Magana, University of Illinois at Chicago; Mary Matteliano, University at Buffalo; Pamela Patt, Shriners Hospitals for Children, Chicago; Amy Rauworth, NCHPAD, Lakeshore Foundation; Mary Segal, Temple University; Debbie Thorpe, University of North Carolina at Chapel Hill; Lee Wallace, University of Tennessee Health Science Center; Glen White, University of Kansas; Angela Odoms-Young, University of Illinois at Chicago. We would also like to acknowledge and thank our CDC consultant David Brown for his time and input.

\section{Funding}

The contents of this publication were developed under a grant from the Department of Education, NIDRR grant number H133A120102. However, those contents do not necessarily represent the policy of the Department of Education, and you should not assume endorsement by the Federal Government.

\section{Author details}

'University of Alabama at Birmingham/Lakeshore Foundation Research Collaborative, 4000 Ridgeway Dr., Birmingham, AL 35209, USA. ${ }^{2}$ Eunice Kennedy Shriver Center/UMass Medical School, 465 Medford St., Charlestown, MA 02129, USA. ${ }^{3}$ Department of Health Sciences, Boston University, 635 Commonwealth Ave., Boston, MA 02215, USA. ${ }^{4}$ University of New Hampshire, 10 West Edge Drive, Suite 101, Durham, NH 03824, USA. ${ }^{5}$ Disability and Health consultant, 800 Highland Trail, Chapel Hill, NC 27516, USA. ${ }^{6}$ College of Applied Health Sciences, Department of Occupational Therapy, University of Illinois at Chicago, 1919 West Taylor, MC 8-11, Chicago, IL 60612, USA. ${ }^{7}$ Department of Epidemiology and Community Medicine, Faculty of Medicine, University of Ottawa; Senior Scientist, Centre for Practice-Changing Research, The Ottawa Hospital Research Institute, 501 Smyth Road, Box 711, Ottawa, ON K1H 8 L6, Canada.
Received: 10 March 2014 Accepted: 22 July 2014

Published: 14 August 2014

\section{References}

1. Steele CA, Kalnins IV, Rossen BE, Biggar DW, Bortolussi JA, Jutai JW: Age-related health risk behaviors of adolescents with physical disabilities. Soz-Praventivmed 2004, 49:132-141.

2. Darrah J, Wessel J, Nearingburg P, O'Connor M: Evaluation of a community fitness program for adolescents with cerebral palsy. Pediatr Phys Ther 1999, 11:18-23.

3. Rimmer JA, Wang E, Yamaki K, Davis B: Documenting Disparities in Obesity and Disability. In FOCUS Technical Brief (24). Austin, TX: SEDL; 2009

4. Evans E, Must A, Anderson SE, Curtin C, Scampini R, Maslin M, Bandini L: Dietary patterns and body mass index in children with autism and typically developing children. Res Autism Dis 2012, 6:399-405.

5. Rimmer J, Yamaki K, Davis B, Wang E, Vogel LC: Obesity and obesity-related secondary conditions in adolescents with Intellectual/Developmental disabilities. J Intell Dis Res 2010, 54:787-294.

6. Kinne S, Patrick DL, Doyle DL: Prevalence of secondary conditions among people with disabilities. Am J Public Health 2004, 94:443-445.

7. Rimmer $\mathrm{JH}$, Chen M-D, Hsieh K: A conceptual model for identifying, preventing and treating secondary conditions in people with disabilities. Phy Ther 2011, 91:1728-1738.

8. Yamaki K, Rimmer JH, Lowry BD, Vogel LC: Prevalence of obesity-related chronic health conditions in overweight adolescents with disabilities. Res Dev Disab 2011, 32:280-288.

9. Carroll D, Courtney-Long EA, Stevens AC, Sloan ML, Lullo C, Visser SN, Fox MH, Armour BS, Campbell VA, Brown DR, Dorn JM: Vital signs: disability and physical activity - United States, 2009-2012. MMWR 2014, 63(early release):p. 1-p. 7.

10. Sit CH, McManus A, McKenzie TL, Lian J: Physical activity levels of children in special schools. Prev Med 2007, 45:424-432

11. Institute of Medicine: Accelerating Progress in Obesity Prevention: Solving the Weight of the Nation. Washington, DC: The National Academies Press; 2012

12. Malone LA, Barfield JP, Brasher JD: Perceived benefits and barriers to exercise among persons with physical disabilities or chronic health conditions within action or maintenance stages of exercise. Dis Health $J$ 2012, 5:254-260

13. Kirchner $C E$, Gerber $E G$, Smith $B C$ : Designed to deter: community barriers to physical activity for people with visual or motor impairments. Am J Prev Med 2008, 34:349-352

14. Murphy N, Carbone P, Council on Children With Disabilities: Promoting the participation of children with disabilities in sports, recreation, and physical activities. Pediatrics 2008, 121:1057-1061.

15. Dunn J, Leitschuh C: Special Physical Education. Dubuque, IA: Kendall/Hunt Publishing; 2006

16. Rimmer JH: The conspicuous absence of people with disabilities in public fitness and recreation facilities: lack of interest or lack of access? Am Health Promot 2005, 19(5):327-329. ii.

17. lezzoni L: Eliminating health and health care disparities among the growing population of people with disabilities. Health Aff 2011, 30:1947-1954

18. Williams AS, Moore SM: Universal design of research: Inclusion of persons with disabilities in mainstream biomedical studies. Sci Trans Med 2011 $3: 1-5$

19. Tomlinson M, Swartz L, Officer A, Chan KY, Rudan I, Saxena S: Research priorities for health of people with disabilities:an expert opinion exercise. Lancet 2009, 374:18571862.

20. Barrable B, Thorogood N, Noonan V, Tomkinson J, Joshi P, Stephenson K, Barclay J, Kovac Burns K: Model for bridging the translational "valleys of death" in spinal cord injury research. J Healthcare Leadership 2014, 6:15-27.

21. Institute of Medicine: Clinical Practice Guidelines We Can Trust. Washington, DC: The National Academies Press; 2011.

22. Harrison M, van den Hoek J, Canadian Guideline Adaptation Study Group: CAN-IMPLEMENT: A Guideline Adaptation and Implementation Planning Resource. Kingston, Ontario, Canada: Queen's University School of Nursing and Canadian Partnership Against Cancer; 2012.

23. Harrison M, Grahan ID, van den Hoek J, Dogherty EJ, Carley ME, Angus V: Guideline adaptation and implementation planning: a prospective observational study. Implement Sci 2013, 8:49-62. 
24. Guidelines International Network: Working Groups/Adaptation. 2013. Available from: http://www.g-i-n.net/activities/adaptation.

25. Fervers B, Burgers JS, Haugh MC, Latreille J, Mlika-Cabbane N, Paquet L, Coulombe M, Poirier M, Burnand B: Adaptation of clinical guidelines: literature review and proposition for a framework and procedure. In J Qual Health Care 2006, 18:167-176.

26. Graham I, Harrison MB, Lorimer K, Piercianowski T, Friedberg E, Buchanan M, Harris C: Adapting national and international leg ulcer practice guidelines for local use: The Ontario Leg Ulcer Community Care Protocol. Adv Skin Wound Care 2005, 18:307-318.

27. Centers for Disease Control and Prevention: Recommended community strategies and measurements to prevent obesity in the United States. MMWR 2009, 58(RR-7):p. 1-p. 30.

28. Blanck H, Collins J: CDC's winnable battles: Improved nutrition, physical activity, and decreased obesity. Child Obes 2013, 9:469-471.

29. Gagliardi A, Brouwers MC: Integrating guideline development and implementation: analysis of guideline development manual instructions for generating implementation advice. Implement Sci 2012, 7:67.

30. Hilbink M, Ouwens $M$, Burgers JS, Kool RB: A new impetus for guideline development and implementation: construction and evaluation of a toolbox. Implement Sci 2014, 9:1-7.

31. Brouwers M, Kho ME, Browman GP, Burgers JS, Cluzeau F, Feder G, Fervers B, Graham ID, Grimshaw J, Hanna SE, Littlejohns P, Makarski J, Zitzelsberger L: AGREE II: advancing guideline development, reporting and evaluation in health care. CMAJ 2010, 182:E834-E842.

32. Drum C, Peterson JJ, Culley C, Krahn G, Heller T, Kimpton T, McCubbin J, Rimmer JH, Seekins T, Suzuki R, White G: Guidelines and Criteria for the implementation of community-based health promotion programs for individuals with disabilities. Am J Health Promot 2009, 24(2):93-101.

33. Abdullah N, Horner-Johnson W, Drum CE, Krahn GL, Staples E, Weisser J, Hammond L: Healthy lifestyles for people with disabilities. Calif J of Health Promot 2004, 2:42-54.

34. Marin G: Defining culturally appropriate community interventions: Hispanics as a case study. J Community Psychol 1993, 21(2):149-161.

35. Suarez-Balcazar $Y$, Frieseman J, Lukyanova V: Culturally competent interventions to address obesity among African American and Latino children and youth. Occup Ther Health Care 2013, 27:113-128.

36. Qaseem A, Forland F, Macbeth F, Ollenschlager G, Phillips S, van der Wees P: Guidelines International Network: toward international standards for clinical practice guidelines. Ann Intern Med 2012, 156:525-531.

37. Rimmer JH, Chen M-D, McCubbin JA, Drum C, Peterson J: Exercise intervention research on persons with disabilities. What we know and where we need to go. Am J Phys Med Rehabil 2010, 89:249-263.

38. Rimmer $\mathrm{JH}$ : Promoting inclusive community-based obesity prevention programs for children and adolescents with disabilities: The why and how. Child Obes 2011, 7:177-184.

39. Steele CA, Kalnins IV, Jutai JW, Stevens SE, Bortolussi JA, Biggar DW: Lifestyle health behaviors of 11- to 16-year old youth with physical disabilities. Health Educ Res 1996, 11:173-186.

40. Drum C, Krahn G, Culley C, Hammond L: Recognizing and responding to the health disparities of people with disabilities. Californian J Health Promot 2005, 3:29-42.

41. Boslaugh S, Andresen EM: Correlates of physical activity for adults with disability. Prev Chron Dis 2006, 3(3):1-14.

\section{Submit your next manuscript to BioMed Central and take full advantage of:}

- Convenient online submission

- Thorough peer review

- No space constraints or color figure charges

- Immediate publication on acceptance

- Inclusion in PubMed, CAS, Scopus and Google Scholar

- Research which is freely available for redistribution

Submit your manuscript at www.biomedcentral.com/submit 